\title{
Atributos físicos do solo em cultivo de cebola sob sistemas de plantio direto e preparo convencional
}

\section{Soil physical attributes of onion cultivation under no-tillage and conventional tillage systems}

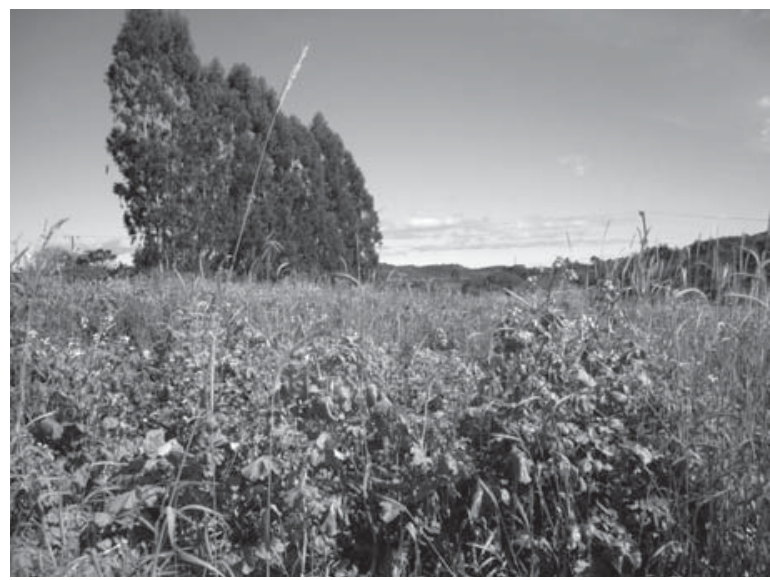

ARCÂNGELO LOSS ${ }^{1,4}$

ELANO DOS SANTOS JUNIOR'

DANIELA SCHMITZ'

MILTON DA VEIGA ${ }^{2}$

CLAUDINEI KURTZ ${ }^{3}$

JUCINEI JOSÉ COMIN'

Plantas de coberturas aos $\mathbf{1 0 0}$ dias após a semeadura.

Foto: M. Souza

\section{RESUMO}

Este trabalho avaliou os atributos físicos do solo cultivado com cebola em sistemas plantio direto de hortaliças (SPDH) e preparo convencional (SPC) do solo, comparados à área de floresta secundária. Os tratamentos constituíram-se da utilização de plantas de cobertura, solteiras e consorciadas em SPDH agroecológico de cebola por seis anos, em blocos ao acaso com cinco repetições: vegetação espontânea, 100\% aveia; 100\% centeio; $100 \%$ nabo forrageiro; consórcio de nabo forrageiro $(14 \%)+$ centeio $(86 \%)$; consórcio de nabo forrageiro $(14 \%)+$ aveia (86\%). Adicionalmente, foram avaliadas duas áreas, uma sob SPC com cultivo de cebola por \pm 38 anos e outra de mata (floresta secundária, \pm 31 anos). Foram coletadas amostras indeformadas de solo nas profundidades de 0-5, 05-10, 10-15 e 15-20 cm, e nestas avaliaram-se a densidade do solo; porosidade total, macro e microporos; diâmetro médio ponderado e geométrico dos agregados, e umidade volumétrica. $\mathrm{O}$ SPDH aumentou os índices de agregação, a porosidade total e umidade volumétrica em comparação ao SPC, e equiparou-se aos índices de agregação em relação à área de mata. O nabo solteiro e consorciado com aveia e centeio melhorou os atributos físicos do solo em comparação com o SPC.

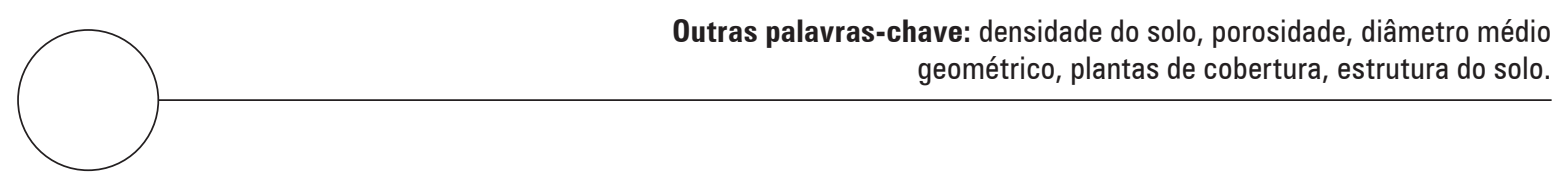

\footnotetext{
1 Departamento de Engenharia Rural, Universidade Federal de Santa Catarina, Programa de Pós-graduação em Agroecossistemas, Florianópolis, SC (Brasil).

2 Universidade do Oeste Catarinense, Campos Novos, SC (Brasil).

3 Pesquisador da Empresa de Pesquisa Agropecuária e Extensão Rural de Santa Catarina, Ituporanga, SC (Brasil).

4 Autor correspondente. arcangeloloss@yahoo.com.br
} 


\section{ABSTRACT}

This study evaluated the physical attributes of Humic Cambisol cultivated with onion in a no-tillage system (NTSV) and conventional tillage system (CTS), as compared to a secondary forest. The treatments consisted of the use of cover crops, alone and intercropped in an agroecological onion NTSV for six years, in randomized blocks with five replications: spontaneous vegetation, $100 \%$ oats; $100 \%$ rye; $100 \%$ wild radish; intercropping of wild radish $(14 \%)$ and rye (86\%); and intercropping of wild radish (14\%) and oats (86\%). We also evaluated an onion cultivation area under CTS for \pm 38 years and a forest area (secondary forest for \pm 31 years). Undisturbed soil samples were collected at depths of $0-5,05-10,10-15$ and $15-20 \mathrm{~cm}$, and the soil density, total porosity, macro and micropores, mean geometric diameter and mean weighted diameter of aggregates, and volumetric moisture were evaluated. The NTSV increased the aggregation indices, total porosity and volumetric moisture in comparison to the CTS and the aggregation indices in relation to the forest area. Alone and intercropped wild radish with oats and rye improved the soil physical attributes as compared to the CTS.

Additional key words: soil density, porosity, geometric mean diameter, cover plants, soil structure.

Data de recepção: 17-12-2016 Aprovado para publicação: 15-04-2017

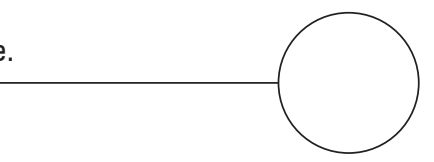

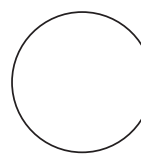

O Estado de Santa Catarina (SC) é o maior produtor nacional de cebola, com uma produção média estimada de 432.000 t para o ano de 2016 (IBGE, 2016). Em SC, o cultivo da cebola ainda se faz, em grande parte, mediante o sistema de preparo convencional do solo (SPC), que caracteriza-se pelo excessivo revolvimento do solo, sendo na ocasião do plantio realizada uma aração e, posteriormente, destorroamento com enxada rotativa, o que ocasiona a pulverização do solo e, consequentemente, sua degradação física, química e biológica (EPAGRI, 2013; Loss et al., 2015).

A redução dos danos ocasionados pelo mau uso do solo pode ser alcançada utilizando-se o sistema plantio direto (SPD), sendo neste caso SPD de hortaliças (SPDH), onde se tem o preparo do solo restrito à linha de plantio. No SPDH de cebola as mudas são produzidas em canteiros e, em seguida, transplantadas em sulcos preparados por semeadoras adaptadas para o corte da cobertura morta e do solo (EPAGRI, 2013).

No SPDH cultivado com cebola, Souza et al. (2013) verificaram que o cultivo e a deposição de plantas de cobertura modificaram os atributos químicos do solo, indicando alterações nos teores de K trocável e $\mathrm{P}$ disponível em função das espécies de plantas de cobertura utilizadas. Os autores ainda relataram que a testemunha (vegetação espontânea) no primeiro ano de estudo (2011), após o acamamento das plantas de cobertura, apresentou os maiores teores de $\mathrm{P}$ e $\mathrm{K}$ em relação aos tratamentos centeio, nabo-forrageiro, nabo+centeio e nabo+cevada. No entanto, a produção média e a produção total da cebola foi superior nos tratamentos com plantas de cobertura nas duas safras avaliadas (2011 e 2012) em comparação com a testemunha.

Conforme o sistema de manejo adotado pode-se ter melhoria, manutenção ou perturbações na estrutura do solo, podendo neste último caso resultar em compactação, que causará problemas na infiltração e na disponibilidade de ar e água para as plantas, além de erosão hídrica do solo (Bronick y Lal, 2005). Por isso, um solo com adequada estrutura apresentará agregados estáveis e melhor distribuição de poros e, por consequência, melhor infiltração de água (Schoenholtz et al., 2000). Atributos da estrutura do solo, como a densidade, a macro e microporosidade, a presença e a estabilidade de agregados, e a resistência à penetração (Luciano et al., 2014; Schoenholtz et al., 2000) devem ser mensurados para avaliar como o manejo influencia as características do solo e a relação dele com a planta (Luciano et al., 2014).

Este trabalho teve como objetivo avaliar os atributos físicos do solo sob SPDH e SPC do solo em comparação com uma área de floresta secundária, em Ituporanga, SC. 


\section{MATERIAL E MÉTODOS}

\section{Descrição da área de estudo}

O estudo foi conduzido na Estação Experimental da Empresa de Pesquisa Agropecuária e Extensão Rural de Santa Catarina (EPAGRI), em Ituporanga, SC (27024'52" S e 49'36'9" W, $475 \mathrm{~m}$ de altitude). O clima da região é subtropical úmido mesotérmico, com temperatura e precipitação média anual de $17,6^{\circ} \mathrm{C}$ e $1.400 \mathrm{~mm}$, respectivamente.

O experimento foi instalado em um Cambissolo Húmico (Embrapa, 2013) sob relevo ondulado (12\% de declividade), apresentando na camada de $0-10 \mathrm{~cm}$ textura franco-argilosa, com 380, 200 e $420 \mathrm{~g} \mathrm{~kg}^{-1}$ de argila, silte e areia, respectivamente, em uma área com histórico de cultivo de cebola em SPC (aração, gradagem e escarificação) por, aproximadamente, 20 anos até 1996. Neste ano foi aplicado calcário na superfície do solo, com posterior incorporação, para elevar o $\mathrm{pH}$ em água até 6,0 . Em seguida, foi implantado o sistema de cultivo mínimo de cebola com rotação de culturas e plantas de cobertura (aveia, mucuna, milheto, crotalaria e ervilhaca), que permaneceu até 2009. Posteriormente, instalou-se o experimento com o SPDH de cebola, mantendo-se um tratamento sob SPC para comparação com o SPDH.

\section{Características dos tratamentos e do delineamento utilizados}

$\mathrm{Na}$ instalação do experimento, em 2009, a vegetação espontânea foi dessecada, usando-se o herbicida glifosato, e a partir de então, não foram mais utilizados agrotóxicos e fertilizantes minerais, caracterizando um sistema de produção agroecológico.

Os tratamentos constituíram-se do plantio de plantas de cobertura solteiras e consorciadas, sendo: (1) testemunha com vegetação espontânea, composta de 20 famílias botânicas, com predomínio de: Amaranthaceae $(10 \%)$, Asteraceae, Caryophyllaceae, Compositae (10\%), Convolvulaceae, Cruciferae, Cyperaceae (25\%), Euphorbiaceae, Fabaceae, Lamiaceae (10\%), Leguminosae, Liliaceae, Malvaceae, Oxalidaceae (10\%), Plantaginaceae, Poaceae, Polygonaceae (20\%), perfazendo 85\% (Vilanova, 2010); (2) 100\% aveia (Avena strigosa), com densidade de semeadura (DS) de $120 \mathrm{~kg} \mathrm{ha}^{-1}$; (3) 100\% centeio (Secale cereale), com DS de $120 \mathrm{~kg} \mathrm{ha}^{-1}$; (4) 100\% nabo-forrageiro (Raphanus sativus), com DS de $20 \mathrm{~kg} \mathrm{ha}^{-1}$; (5) consórcio de nabo-forrageiro (14\%) e centeio (86\%), com DS de 10 e $60 \mathrm{~kg} \mathrm{ha}^{-1}$, respectivamente e (6) consórcio de naboforrageiro (14\%) e aveia (86\%), com DS de 10 e $60 \mathrm{~kg}$ ha $^{-1}$, respectivamente.

As quantidades de sementes por hectare foram calculadas com base na recomendação de Monegat (1991), sendo acrescidos de $50 \%$ sobre a quantidade calculada. As espécies de inverno foram semeadas a lanço em abril de cada ano (2009 a 2014) e, em seguida, uma máquina semeadora de cereais foi passada duas vezes na área. Em julho de cada ano (2009 a 2014), todas as espécies de inverno foram acamadas por meio de um rolo-faca modelo RF240 (MBO Ltda).

Os tratamentos foram dispostos em blocos ao acaso, com cinco repetições. Cada unidade experimental tinha $25 \mathrm{~m}^{2}(5 \times 5 \mathrm{~m})$. Adicionalmente, foram avaliados mais dois tratamentos, sendo um a área original de cultivo de cebola mantida sob SPC por 38 anos até 2014. No SPC, a partir do ano de 2007, a cebola foi cultivada em sucessão com milheto no verão, que foi acamado na floração com rolo-faca e, após 30-60 d, realizada aração seguida de gradagem antes do transplantio das mudas de cebola. O outro tratamento adicional, uma floresta secundária com \pm 31 anos sobre a mesma classe de solo, se encontrava a $\pm 500 \mathrm{~m}$ de distância do experimento, representou a condição natural do solo.

A adubação no SPC foi realizada conforme recomendações da COFS-RS/SC (2004), sendo aplicados 165 $\mathrm{kg} \mathrm{ha}^{-1}$ de $\mathrm{P}_{2} \mathrm{O}_{5}$ (superfosfato triplo), $105 \mathrm{~kg} \mathrm{ha}^{-1}$ de $\mathrm{K}_{2} \mathrm{O}$ (cloreto de potássio) e $192 \mathrm{~kg} \mathrm{ha}^{-1}$ de $\mathrm{N}$ (nitrato de amônio). Em 2010 o SPC recebeu calagem para elevação do $\mathrm{pH}$ para 6,0 pelo método SMP (COFS-RS/ SC, 2004).

No SPDH, após o acamamento das plantas de cobertura de inverno, no mês de julho de cada ano, foram aplicados na área $96 \mathrm{~kg} \mathrm{ha}^{-1}$ de $\mathrm{P}_{2} \mathrm{O}_{5}$, na forma de fosfato natural de Gafsa moído, $175 \mathrm{~kg} \mathrm{ha}^{-1}$ de $\mathrm{P}_{2} \mathrm{O}_{5}, 125$ $\mathrm{kg} \mathrm{ha}^{-1}$ de $\mathrm{K}_{2} \mathrm{O}$ e $160 \mathrm{~kg} \mathrm{ha}^{-1}$ de $\mathrm{N}$, na forma de dejetos de aves, sendo metade aplicada no plantio das mudas de cebola e o restante $30 \mathrm{~d}$ após o plantio. A partir da safra de 2011, não foi aplicado fosfato natural, pois os teores de $\mathrm{P}$ foram interpretados como muito altos (COFS-SC/RS, 2004). Em seguida, foram abertos sulcos usando uma máquina de plantio direto adaptada e foram transplantadas manualmente as mudas de cebola cv. Empasc 352 - Bola Precoce, procedimento também usado no SPC. O espaçamento foi de $0,50 \mathrm{~m}$ 
nas entrelinhas e 0,10 m entre plantas, com 10 linhas de cebola por parcela.

No SPDH foram realizadas capinas aos 60 e 90 d após o plantio das mudas de cebola. Após a colheita da cebola, em dezembro de cada ano, sempre foi realizado o plantio da mucuna-preta no verão em toda a área cultivada, na DS de $120 \mathrm{~kg} \mathrm{ha}{ }^{-1}$. A mucuna é acamada no mês de março de cada ano para em abril se fazer a semeadura das plantas de cobertura de inverno.

\section{Coleta e preparação das amostras de solo para as análises físicas}

Após 6 anos de implantação dos tratamentos (20092014) foi realizada uma coleta de amostras de solo indeformadas (com estrutura preservada) em todos os tratamentos, seguindo-se a metodologia preconizada por Veiga (2011). Para tal, foram abertas mini trincheiras de $40 \times 40 \times 40 \mathrm{~cm}$ e coletadas amostras com o auxílio de anéis metálicos de $5 \mathrm{~cm}$ de diâmetro por $5 \mathrm{~cm}$ de altura em quatro profundidades $(0-5,5-10$, 10-15 e 15-20 cm). Em seguida as amostras foram acondicionadas em sacos plásticos e encaminhadas ao Laboratório. Todas as análises físicas seguiram o protocolo descrito por Embrapa (1997) e Veiga (2011). No laboratório, as amostras foram preparadas para seguir uma bateria de análises. Num primeiro momento elas foram saturadas por capilaridade, sendo dispostas em um recipiente com água até atingir metade da altura do anel metálico e deixar-se nessa condição por $24 \mathrm{~h}$ para que toda a amostra fosse saturada. Em seguida foi aferido a massa das amostras saturadas.

Para a obtenção da curva de retenção de água no solo, as amostras saturadas foram dispostas sobre uma mesa de tensão aplicando-se tensões de 0,6 e 6 kPa, passando em seguida para o extrator de Richards, com aplicação de tensões de 60 e $600 \mathrm{kPa}$. Ao fim da aplicação de cada tensão foi aferido a massa da amostra, passando então para a tensão seguinte. A distribuição de tamanho de poros foi obtida através dos dados da curva de retenção de água, considerando-se que os macroporos, com diâmetro maior ou igual a $50 \mu \mathrm{m}$ retém água do solo saturado até a tensão de $6 \mathrm{kPa}$, e os poros com diâmetro menor do que $5 \mu \mathrm{m}$ (microporos) retém a água até a tensão de $600 \mathrm{kPa}$.

$\mathrm{Na}$ sequência foi determinada a estabilidade dos agregados do solo retirando-se uma amostra do anel metálico que foi destorroada até que toda a massa de solo passasse por uma malha de $8 \mathrm{~mm}$ e os agregados foram então secos ao ar até que a umidade se estabilizasse. Posteriormente, o solo foi peneirado em malhas de 4; 2; 1 e 0,5 mm e fundo, sendo os agregados retidos em cada malha, posteriormente pesados, compondo os dados de tamanho dos agregados. Para a distribuição de tamanho dos agregados estáveis em água, os agregados foram colocados sobre as malhas das peneiras com os mesmos diâmetros da determinação anterior e, em seguida, as peneiras foram posicionadas no interior do aparelho e adicionou-se água até que esta tocasse o fundo da malha superior, deixando nessa condição por 10 min. Em seguida, o aparelho de peneiramento foi acionado por mais $10 \mathrm{~min}$, promovendo uma agitação branda. Cada classe de agregados foi quantificada após a secagem em estufa a $105^{\circ} \mathrm{C}$.

Com as massas dos agregados retidos nas classes de $\varnothing:<0,5 ; 0,5-1,0 ; 1,0-2,0$ e 2,0-4,0 mm e o $\varnothing$ médio da respectiva classe, determinou-se o $\varnothing$ médio ponderado aritmético e geométrico dos agregados secos ao ar (DMAsa e DMGsa, respectivamente) e estáveis em água (DMAea e DMGea, respectivamente), bem como o índice de estabilidade de agregados (IEA ${ }_{D M A}=$ DMAea/DMAsa e IEA $\left.{ }_{\text {DMG }}=\mathrm{DMGea} / \mathrm{DMGsa}\right)$, conforme Embrapa (1997) e Veiga (2011).

\section{Análises estatísticas}

Os resultados foram analisados quanto à normalidade e homogeneidade dos dados por meio dos testes de Lilliefors e Bartlet. Posteriormente, os dados foram submetidos à análise de variância com aplicação do teste $\mathrm{F}$ e os valores médios, quando significativos, comparados entre si pelo teste Skott-Knott a 5\%.

\section{RESULTADOS E DISCUSSÃO}

Os menores valores de densidade do solo (Ds) foram encontrados na área de mata, em todas as profundidades, em comparação ao SPDH e o SPC. Não foram verificadas diferenças entre o SPDH e o SPC na camada de 0-5 e $5-10 \mathrm{~cm}$, mas para $10-15$ e $15-20 \mathrm{~cm}$, o SPC apresentou os menores valores de Ds (Tab. 1). Os menores valores de Ds na área de mata são decorrentes da ausência de interferência antrópica, além dos maiores teores de COT que variam de 52,83 g $\mathrm{kg}^{-1}(0-5 \mathrm{~cm})$ a $26,70 \mathrm{~g} \mathrm{~kg}^{-1}(10-20 \mathrm{~cm})$ em comparação às demais áreas, com valores médios variando de $28,00 \mathrm{~g} \mathrm{~kg}^{-1}(0-5 \mathrm{~cm})$ a $18,00 \mathrm{~g} \mathrm{~kg}^{-1}(10-20 \mathrm{~cm})$ Loss et al., 2015). Já no SPC, os menores valores de Ds em profundidade são decorrentes das práticas de aração 


\begin{tabular}{|c|c|c|c|c|c|c|c|}
\hline \multirow{3}{*}{ Tratamentos } & Ds & $\mathrm{DMAA}_{\mathrm{SA}}$ & $\mathrm{DMA}_{\mathrm{EA}}$ & IEA & $\mathrm{DMG}_{\mathrm{SA}}$ & $\mathrm{DMG}_{\mathrm{EA}}$ & IEA \\
\hline & $\mathrm{Mg} \mathrm{m}^{-3}$ & $\mathrm{~mm}$ & $\mathrm{~mm}$ & - & $\mathrm{mm}$ & $\mathrm{mm}$ & - \\
\hline & \multicolumn{7}{|c|}{$0-5 \mathrm{~cm}$} \\
\hline Aveia & 1,28 a & $3,278 \mathrm{a}$ & $1,520 \mathrm{a}$ & $0,46 \mathrm{a}$ & $2,218 \mathrm{a}$ & $0,778 \mathrm{a}$ & $0,35 \mathrm{a}$ \\
\hline Centeio & $1,32 \mathrm{a}$ & $3,244 \mathrm{a}$ & $1,612 \mathrm{a}$ & $0,49 a$ & $2,150 \mathrm{a}$ & $0,746 \mathrm{a}$ & $0,34 \mathrm{a}$ \\
\hline Nabo & $1,34 \mathrm{a}$ & $3,200 \mathrm{a}$ & $1,652 \mathrm{a}$ & $0,51 \mathrm{a}$ & $2,158 \mathrm{a}$ & $0,820 \mathrm{a}$ & $0,38 \mathrm{a}$ \\
\hline Centeio + nabo & $1,39 a$ & $3,100 \mathrm{a}$ & $1,746 \mathrm{a}$ & $0,56 \mathrm{a}$ & $2,018 \mathrm{a}$ & $0,832 \mathrm{a}$ & $0,41 \mathrm{a}$ \\
\hline Aveia + nabo & $1,28 \mathrm{a}$ & $3,122 \mathrm{a}$ & $1,714 \mathrm{a}$ & $0,54 a$ & $2,082 \mathrm{a}$ & 0,868 a & $0,42 \mathrm{a}$ \\
\hline Testemunha & $1,32 \mathrm{a}$ & $3,176 \mathrm{a}$ & $1,892 \mathrm{a}$ & $0,59 \mathrm{a}$ & $2,052 \mathrm{a}$ & 0,968 a & $0,47 \mathrm{a}$ \\
\hline SPC & $1,40 \mathrm{a}$ & $3,320 \mathrm{a}$ & $0,718 \mathrm{c}$ & $0,22 b$ & $2,246 \mathrm{a}$ & $0,370 \mathrm{~b}$ & $0,16 b$ \\
\hline Mata & $0,94 \mathrm{~b}$ & $2,684 b$ & $1,254 b$ & $0,46 \mathrm{a}$ & $1,630 b$ & 0,706 a & $0,43 \mathrm{a}$ \\
\hline CV\% & 10,24 & 7,20 & 22,66 & 22,33 & 10,88 & 25,84 & 25,46 \\
\hline \multicolumn{8}{|c|}{$5-10 \mathrm{~cm}$} \\
\hline Aveia & $1,51 \mathrm{a}$ & 3,236 a & $1,090 \mathrm{~b}$ & $0,34 \mathrm{a}$ & $2,218 \mathrm{a}$ & $0,558 \mathrm{a}$ & $0,26 \mathrm{a}$ \\
\hline Centeio & $1,48 \mathrm{a}$ & $3,384 \mathrm{a}$ & $1,326 \mathrm{a}$ & $0,39 a$ & $2,160 \mathrm{a}$ & $0,642 \mathrm{a}$ & $0,29 a$ \\
\hline Nabo & $1,49 \mathrm{a}$ & $3,212 \mathrm{a}$ & $1,140 \mathrm{~b}$ & $0,35 \mathrm{a}$ & $2,108 \mathrm{a}$ & $0,576 \mathrm{a}$ & $0,27 \mathrm{a}$ \\
\hline Centeio + nabo & $1,50 \mathrm{a}$ & $3,404 \mathrm{a}$ & $1,564 \mathrm{a}$ & $0,46 \mathrm{a}$ & $2,298 \mathrm{a}$ & $0,702 \mathrm{a}$ & $0,31 \mathrm{a}$ \\
\hline Aveia + nabo & $1,48 \mathrm{a}$ & $3,382 \mathrm{a}$ & $1,228 b$ & 0,36 a & $2,312 \mathrm{a}$ & $0,642 \mathrm{a}$ & $0,28 \mathrm{a}$ \\
\hline Testemunha & $1,52 \mathrm{a}$ & $3,284 \mathrm{a}$ & $1,078 \mathrm{~b}$ & $0,32 \mathrm{a}$ & $2,196 \mathrm{a}$ & $0,540 \mathrm{a}$ & $0,25 \mathrm{a}$ \\
\hline SPC & $1,45 a$ & $3,354 \mathrm{a}$ & $0,832 \mathrm{c}$ & $0,25 b$ & $2,304 \mathrm{a}$ & $0,410 \mathrm{~b}$ & $0,18 b$ \\
\hline Mata & $0,95 b$ & $2,662 \mathrm{~b}$ & $1,082 b$ & $0,41 \mathrm{a}$ & $1,672 \mathrm{~b}$ & $0,550 \mathrm{a}$ & $0,33 \mathrm{a}$ \\
\hline CV\% & 4,77 & 8,76 & 12,26 & 22,8 & 14,48 & 17,87 & 22,56 \\
\hline \multicolumn{8}{|c|}{$10-15 \mathrm{~cm}$} \\
\hline Aveia & $1,52 \mathrm{a}$ & $3,300 \mathrm{a}$ & $0,750 \mathrm{c}$ & $0,22 b$ & $2,192 \mathrm{a}$ & $0,424 b$ & $0,19 b$ \\
\hline Centeio & $1,53 \mathrm{a}$ & $3,292 \mathrm{a}$ & $0,942 b$ & $0,34 \mathrm{~b}$ & $2,200 \mathrm{a}$ & $0,472 b$ & $0,21 \mathrm{~b}$ \\
\hline Nabo & $1,53 \mathrm{a}$ & $3,204 \mathrm{a}$ & $1,086 b$ & $0,34 \mathrm{~b}$ & $2,104 \mathrm{a}$ & $0,542 b$ & $0,26 \mathrm{~b}$ \\
\hline Centeio + nabo & $1,49 a$ & $3,126 \mathrm{a}$ & $1,012 b$ & $0,32 \mathrm{~b}$ & $2,006 \mathrm{a}$ & $0,506 \mathrm{~b}$ & $0,25 \mathrm{~b}$ \\
\hline Aveia + nabo & $1,53 \mathrm{a}$ & 3,326 a & $0,932 \mathrm{~b}$ & $0,28 \mathrm{~b}$ & $2,208 \mathrm{a}$ & $0,496 \mathrm{~b}$ & $0,22 b$ \\
\hline Testemunha & $1,53 \mathrm{a}$ & $3,320 \mathrm{a}$ & $0,794 \mathrm{c}$ & $0,23 \mathrm{~b}$ & $2,200 \mathrm{a}$ & $0,446 b$ & $0,20 \mathrm{~b}$ \\
\hline SPC & $1,42 b$ & $3,334 \mathrm{a}$ & $0,798 \mathrm{c}$ & $0,24 \mathrm{~b}$ & $2,310 \mathrm{a}$ & $0,400 \mathrm{~b}$ & $0,17 \mathrm{~b}$ \\
\hline Mata & $1,02 \mathrm{c}$ & $2,616 \mathrm{~b}$ & $1,592 \mathrm{a}$ & $0,61 \mathrm{a}$ & $1,610 b$ & $0,884 \mathrm{a}$ & $0,55 \mathrm{a}$ \\
\hline CV\% & 4,02 & 6,55 & 15,96 & 26,06 & 9,88 & 24,55 & 25,43 \\
\hline \multicolumn{8}{|c|}{$15-20 \mathrm{~cm}$} \\
\hline Aveia & $1,50 \mathrm{a}$ & $3,108 \mathrm{a}$ & $0,540 \mathrm{c}$ & $0,17 \mathrm{c}$ & $2,090 \mathrm{a}$ & $0,326 \mathrm{c}$ & $0,16^{\mathrm{ns}}$ \\
\hline Centeio & $1,50 \mathrm{a}$ & $3,140 \mathrm{a}$ & $0,652 \mathrm{c}$ & $0,21 \mathrm{c}$ & $2,100 \mathrm{a}$ & $0,382 \mathrm{c}$ & 0,18 \\
\hline Nabo & $1,53 \mathrm{a}$ & $3,300 \mathrm{a}$ & $1,022 b$ & $0,31 \mathrm{~b}$ & $2,192 \mathrm{a}$ & $0,498 \mathrm{~b}$ & 0,23 \\
\hline Centeio + nabo & $1,54 \mathrm{a}$ & $3,334 \mathrm{a}$ & $0,852 \mathrm{c}$ & $0,26 \mathrm{c}$ & $2,234 \mathrm{a}$ & $0,456 b$ & 0,21 \\
\hline Aveia + nabo & $1,52 \mathrm{a}$ & $3,228 \mathrm{a}$ & $0,674 \mathrm{c}$ & $0,21 \mathrm{c}$ & $2,100 \mathrm{a}$ & $0,374 \mathrm{c}$ & 0,19 \\
\hline Testemunha & $1,52 \mathrm{a}$ & $3,210 \mathrm{a}$ & $0,606 \mathrm{c}$ & $0,19 \mathrm{c}$ & $2,150 \mathrm{a}$ & $0,374 \mathrm{c}$ & 0,17 \\
\hline SPC & $1,37 \mathrm{~b}$ & $3,540 \mathrm{a}$ & $0,624 \mathrm{c}$ & $0,18 \mathrm{c}$ & $2,576 \mathrm{a}$ & $0,364 \mathrm{c}$ & 0,14 \\
\hline Mata & $0,96 \mathrm{c}$ & $2,686 \mathrm{~b}$ & $1,330 \mathrm{a}$ & $0,49 a$ & $1,710 \mathrm{~b}$ & $0,744 \mathrm{a}$ & 0,44 \\
\hline CV\% & 5,03 & 11,38 & 29,31 & 27,78 & 11,82 & 25,73 & 36,64 \\
\hline
\end{tabular}

Médias seguidas de mesma letra na coluna não diferem entre si (Skott-Knott $P \leq 0,05)$; CV: coeficiente de variação. 
e gradagem, que ocasionam a inversão das camadas superficiais para maiores profundidades, corroborando com a maior macroporosidade na profundidade de 10-15 cm (Tab. 2).

Para os valores de diâmetro médio aritmético e geométrico secos ao ar (DMAsa e DMGsa, respectivamente), os menores valores foram verificados na mata e os maiores no SPDH e SPC, sem diferenças entre esses tratamentos (Tab. 1). O tamisamento via seco parece romper grande parte das "forças de coesão" entre as partículas, de tal forma que durante a agitação eletromagnética não é possível diferenciar agregados formados recentemente daqueles estáveis ou que sofreram um processo de estabilização (Ramos et al., 2014), o que explica a inexistência de diferenças entre o SPDH e SPC.

Para os índices diâmetro médio aritmético e geométrico estáveis em água (DMAea e DMGea, respectivamente), verificaram-se menores valores no SPC nas camadas de $0-5$ e $5-10 \mathrm{~cm}$. Para o DMAea, os tratamentos centeio e centeio + nabo $(5-10 \mathrm{~cm})$, centeio, nabo, centeio + nabo e aveia + nabo (10$15 \mathrm{~cm})$ e somente nabo $(15-20 \mathrm{~cm})$, apresentaram maiores valores no SPDH em comparação aos demais tratamentos e ao SPC. Para o DMGea, apenas para $15-20 \mathrm{~cm}$, os tratamentos com nabo e centeio + nabo apresentaram maiores valores entre os tratamentos em SPDH e o SPC (Tab. 1). Os maiores valores de DMAea e DMGea no tratamento com o nabo-forrageiro podem ser em razão do sistema radicular pivotante e agressivo dessa crucífera que, à medida que se desenvolve, pressiona as partículas de solo ao seu redor, favorecendo a formação de agregados maiores e mais resistentes (Casali, 2012; Guedes Filho et al., 2013).

Os maiores valores de DMGea e DMAea para o $\mathrm{SPDH}$ em relação ao $\mathrm{SPC}$ e à mata $(0-5 \mathrm{~cm}$ para DMAea) podem ser decorrentes da exsudação de polissacarídeos pelas plantas de cobertura. Os polissacarídeos exsudados de plantas de azevém, centeio e cevada têm, em um curto espaço de tempo, alto efeito aglutinador das partículas do solo, com ênfase para solos que foram submetidos a sistemas de cultivo intensivos durante longo períodos (Liu et al., 2005), a exemplo do SPC por 38 anos, neste estudo.

Além dos efeitos já mencionados, o favorecimento da formação de agregados, evidenciado pelos maiores índices de DMAea e DMGea, no consórcio do centeio + nabo decorre do fato de o centeio apresentar
Tabela 2. Valores médios dos atributos relacionados à porosidade do solo.

\begin{tabular}{|c|c|c|c|}
\hline \multirow{2}{*}{ Tratamentos } & PT & MAP & MIP \\
\hline & \multicolumn{3}{|c|}{$\mathrm{cm}^{3} \mathrm{~cm}^{-3}$} \\
\hline \multicolumn{4}{|c|}{$0-5 \mathrm{~cm}$} \\
\hline Aveia & $0,57 \mathrm{~b}$ & $0,21 \mathrm{~ns}$ & $0,36 \mathrm{~ns}$ \\
\hline Centeio & $0,52 \mathrm{c}$ & 0,22 & 0,30 \\
\hline Nabo & $0,50 \mathrm{c}$ & 0,20 & 0,30 \\
\hline Centeio + nabo & $0,49 \mathrm{c}$ & 0,20 & 0,29 \\
\hline Aveia + nabo & $0,54 \mathrm{c}$ & 0,22 & 0,32 \\
\hline Testemunha & $0,49 \mathrm{c}$ & 0,21 & 0,28 \\
\hline SPC & $0,44 \mathrm{~d}$ & 0,20 & 0,24 \\
\hline Mata & $0,67 \mathrm{a}$ & 0,30 & 0,37 \\
\hline CV\% & 8,80 & 21,25 & 17,07 \\
\hline \multicolumn{4}{|c|}{$5-10 \mathrm{~cm}$} \\
\hline Aveia & $0,45 b$ & $0,15 \mathrm{~ns}$ & $0,30 \mathrm{~ns}$ \\
\hline Centeio & $0,45 b$ & 0,16 & 0,29 \\
\hline Nabo & $0,46 b$ & 0,17 & 0,29 \\
\hline Centeio + nabo & $0,45 a$ & 0,16 & 0,29 \\
\hline Aveia + nabo & $0,47 \mathrm{~b}$ & 0,18 & 0,29 \\
\hline Testemunha & $0,46 \mathrm{~b}$ & 0,16 & 0,30 \\
\hline SPC & $0,49 \mathrm{~b}$ & 0,21 & 0,28 \\
\hline Mata & $0,62 \mathrm{a}$ & 0,24 & 0,38 \\
\hline CV\% & 5,97 & 15,58 & 8,76 \\
\hline \multicolumn{4}{|c|}{$10-15 \mathrm{~cm}$} \\
\hline Aveia & $0,43 b$ & $0,15 \mathrm{c}$ & $0,28 \mathrm{~ns}$ \\
\hline Centeio & $0,46 \mathrm{~b}$ & $0,16 \mathrm{c}$ & 0,30 \\
\hline Nabo & $0,44 \mathrm{~b}$ & $0,15 c$ & 0,29 \\
\hline Centeio + nabo & $0,47 \mathrm{~b}$ & $0,15 \mathrm{c}$ & 0,32 \\
\hline Aveia + nabo & $0,45 b$ & $0,16 \mathrm{c}$ & 0,29 \\
\hline Testemunha & $0,44 \mathrm{~b}$ & $0,15 c$ & 0,29 \\
\hline SPC & $0,48 b$ & $0,19 b$ & 0,29 \\
\hline Mata & $0,61 \mathrm{a}$ & $0,23 \mathrm{a}$ & 0,38 \\
\hline CV\% & 9,65 & 15,33 & 12,34 \\
\hline \multicolumn{4}{|c|}{$15-20 \mathrm{~cm}$} \\
\hline Aveia & $0,45 \mathrm{~ns}$ & $0,15 \mathrm{~ns}$ & $0,30 \mathrm{~ns}$ \\
\hline Centeio & 0,46 & 0,16 & 0,30 \\
\hline Nabo & 0,45 & 0,15 & 0,30 \\
\hline Centeio + nabo & 0,44 & 0,16 & 0,28 \\
\hline Aveia + nabo & 0,44 & 0,17 & 0,27 \\
\hline Testemunha & 0,43 & 0,14 & 0,29 \\
\hline SPC & 0,49 & 0,20 & 0,29 \\
\hline Mata & 0,59 & 0,27 & 0,32 \\
\hline CV\% & 7,56 & 18,94 & 12,82 \\
\hline
\end{tabular}

Médias seguidas de mesma letra na coluna não diferem entre si (Skott-Knott $P \leq 0,05)$; ns: não significativo pelo teste $\mathrm{F}$ a 5\%; CV: coeficiente de variação. 
efeito químico decorrente do sistema radicular fasciculado e denso, distribuindo melhor os exsudatos radiculares; e o nabo promove um efeito físico de compressão do solo (Casali, 2012). Na testemunha, a diversidade que compõe a vegetação espontânea (20 famílias) também favorece os processos físicoquímicos relacionados à agregação, além dos benefícios oriundos do SPD, o que explica a inexistência de diferenças com os demais tratamentos em SPDH na camada de $0-5 \mathrm{~cm}$.

Os índices de estabilidades de agregados para o DMA (IEA $\left.A_{D M A}\right)$ e DMG (IEA $\left.A_{D M G}\right)$ apresentaram comportamento semelhante nas três primeiras camadas, sendo os menores valores verificados no SPC, e ausência de diferenças entre o SPDH e a mata para as profundidades de $0-5$ e $5-10 \mathrm{~cm}$. Em $10-15 \mathrm{~cm}$, apenas o DMG (IEA $\left.\mathrm{DMG}_{\mathrm{DG}}\right)$ da área de mata diferiu dos demais tratamentos, apresentando os maiores valores. $\mathrm{Na}$ camada de $15-20 \mathrm{~cm}$, não foram verificadas diferenças para o IEA $\mathrm{DMG}_{\mathrm{DMG}}$, porém para o $\mathrm{IEA}_{\mathrm{DMA}}$, entre o SPDH e o SPC, o tratamento com nabo solteiro apresentou o maior valor (Tab. 1). Os menores valores de IEA no SPC indicam que este sistema desfavorece a formação de agregados estáveis e no SPDH, ao contrário, tem-se um ambiente edáfico favorável à formação de agregados estáveis, devido ao não revolvimento do solo e à constante presença de raízes e biomassa vegetal oriundas das plantas de cobertura.

A porosidade total (PT), em geral, foi maior na mata, sendo que na camada de $0-5 \mathrm{~cm}$, o SPC apresentou os menores valores em comparação ao SPDH. Neste sistema, o tratamento aveia apresentou os maiores valores de PT (Tab. 2), dados que corroboram com o maior valor de umidade volumétrica nessa mesma camada (Tabela 3). Para as profundidades de 5-10 e $10-15 \mathrm{~cm}$ não foram verificadas diferenças entre o SPC e o SPDH (Tab. 2). Os maiores valores de PT no $\mathrm{SPDH}$ em comparação ao SPC para $0-5 \mathrm{~cm}$ indicam que as plantas de cobertura proporcionam maior $\mathrm{PT}$ no solo devido à constante presença de raízes associada ao não revolvimento do solo, o que acarreta em maiores índices de agregação (Tab. 1), com espaços de vazios entre eles (poros). Além disso, a cobertura vegetal favorece a biota do solo, que por sua vez, corrobora com a maior porosidade na camada superficial do solo.
Para a macroporosidade (MAP) somente foram verificadas diferenças em 10-15 cm, onde os maiores valores ocorreram na mata, seguida por SPC e menores valores no SPDH. Já a microporosidade (MIP) não apresentou diferenças em nenhuma das profundidades (Tab. 2). Os valores de MAP em todos os tratamentos se mostraram adequados para o bom desenvolvimento das raízes e fluxos de água e ar no solo, permanecendo acima do nível crítico de 0,10 $\mathrm{m}^{3} \mathrm{~m}^{-3}$ (Richart et al., 2005; Silva et al., 2006).

A umidade volumétrica (UV) apresentou diferenças entre os tratamentos nas profundidades 0-5 e 10-15 $\mathrm{cm}$ quando aplicadas tensões de 0 e $0,6 \mathrm{kPa}$, com maiores médias na área de mata em ambos os casos. Para 0-5 cm, entre o SPDH e o SPC, os tratamentos aveia, centeio, nabo e aveia + nabo apresentaram os maiores valores para UV 0,0 kPa e, na UV 0,6 kPa, a aveia, o centeio e a aveia + nabo apresentaram os maiores valores em relação aos demais tratamentos. Verificou-se diferenças em 0-5 e 5-10 cm quando foi aplicada a tensão de $6 \mathrm{kPA}$, enquanto em 60 e 600 $\mathrm{kPa}$ as médias não diferiram. Em 10-15 cm, o SPDH e SPC não diferiram entre si nas tensões de 0,0 e 0,6 $\mathrm{KPa}$ (Tab. 3).

$\mathrm{Na}$ tensão de $60 \mathrm{KPa}$, nas profundidades de 0-5 e 5-10 $\mathrm{cm}$, apenas o SPC diferiu dos demais tratamentos, apresentando os menores valores de UV. Na maior tensão $(600 \mathrm{KPa})$, somente foram verificadas diferenças na camada de $0-5 \mathrm{~cm}$, onde o SPC também apresentou os menores valores de UV, e os tratamentos aveia e aveia + nabo os maiores valores (Tab. 3).

Os menores valores de UV no SPC (Tab. 3) corroboram os menores índices de agregação encontrados neste sistema (Tab. 1), indicando o desfavorecimento à manutenção de agregados estáveis, com menor PT na camada superficial e, consequentemente, menor conteúdo de UV. Em contrapartida, os maiores índices de agregação, porosidade total e UV 60 e 600 $\mathrm{kPa}$ nos tratamentos no SPDH em comparação ao SPC indicam melhoria da qualidade física do solo com o SPD de hortaliças com plantas de coberturas, que favorecem a exploração do solo via sistema radicular e ausência de revolvimento do solo, o que não ocasiona a ruptura dos agregados e diminuição da porosidade e menor armazenamento de água, como observado no SPC. 
Tabela 3. Valores médios de umidade volumétrica nas diferentes tensões aplicadas no solo.

\begin{tabular}{|c|c|c|c|c|c|c|c|c|}
\hline \multirow{3}{*}{ Tratamentos } & \multicolumn{8}{|c|}{ Profundidade $(\mathrm{cm})$} \\
\hline & \multicolumn{2}{|c|}{$0-5$} & \multicolumn{2}{|c|}{$5-10$} & \multicolumn{2}{|c|}{$10-15$} & \multicolumn{2}{|c|}{$15-20$} \\
\hline & $0 \mathrm{KPa}$ & $0,6 \mathrm{KPa}$ & $0 \mathrm{KPa}$ & $0,6 \mathrm{KPa}$ & $0 \mathrm{KPa}$ & $0,6 \mathrm{KPa}$ & $0 \mathrm{KPa}$ & $0,6 \mathrm{KPa}$ \\
\hline Aveia & $0,56 \mathrm{~b}$ & $0,48 b$ & $0,45^{\mathrm{ns}}$ & $0,39^{\text {ns }}$ & $0,43 b$ & $0,37 \mathrm{~b}$ & $0,37^{\text {ns }}$ & $0,44^{\text {ns }}$ \\
\hline Centeio & $0,54 \mathrm{~b}$ & $0,44 \mathrm{c}$ & 0,46 & 0,39 & $0,46 b$ & $0,38 \mathrm{~b}$ & 0,38 & 0,46 \\
\hline Nabo & $0,50 \mathrm{~b}$ & $0,41 d$ & 0,47 & 0,40 & $0,45 b$ & $0,38 \mathrm{~b}$ & 0,38 & 0,44 \\
\hline Centeio + nabo & $0,46 \mathrm{c}$ & $0,39 d$ & 0,45 & 0,38 & $0,47 b$ & $0,39 \mathrm{~b}$ & 0,36 & 0,44 \\
\hline Aveia + nabo & $0,54 \mathrm{~b}$ & $0,45 c$ & 0,47 & 0,39 & $0,45 b$ & $0,37 \mathrm{~b}$ & 0,36 & 0,44 \\
\hline Testemunha & $0,46 \mathrm{c}$ & $0,38 d$ & 0,46 & 0,39 & $0,44 \mathrm{~b}$ & $0,37 \mathrm{~b}$ & 0,37 & 0,44 \\
\hline SPC & $0,46 \mathrm{c}$ & $0,37 d$ & 0,48 & 0,39 & $0,49 \mathrm{~b}$ & $0,40 \mathrm{~b}$ & 0,39 & 0,49 \\
\hline Mata & $0,67 \mathrm{a}$ & $0,56 \mathrm{a}$ & 0,62 & 0,54 & $0,61 \mathrm{a}$ & $0,54 \mathrm{a}$ & 0,50 & 0,58 \\
\hline \multirow[t]{2}{*}{ CV\% } & 8,80 & 9,52 & 5,97 & 5,38 & 9,65 & 10,85 & 8,49 & 7,56 \\
\hline & $6 \mathrm{KPa}$ & $60 \mathrm{KPa}$ & $6 \mathrm{KPa}$ & $60 \mathrm{Kpa}$ & $6 \mathrm{KPa}$ & $60 \mathrm{Kpa}$ & $6 \mathrm{KPa}$ & $60 \mathrm{Kpa}$ \\
\hline Aveia & $0,42 \mathrm{~b}$ & $0,39 a$ & $0,35 b$ & 0,33 a & $0,35^{\mathrm{ns}}$ & $0,32^{\mathrm{ns}}$ & $0,34^{\mathrm{ns}}$ & $0,32^{\mathrm{ns}}$ \\
\hline Centeio & $0,38 \mathrm{~b}$ & 0,36 a & $0,35 b$ & $0,33 \mathrm{a}$ & 0,35 & 0,33 & 0,35 & 0,32 \\
\hline Nabo & $0,35 b$ & $0,33 \mathrm{a}$ & $0,35 b$ & $0,32 \mathrm{a}$ & 0,34 & 0,32 & 0,35 & 0,33 \\
\hline Centeio + nabo & $0,33 \mathrm{~b}$ & $0,31 \mathrm{a}$ & $0,34 \mathrm{~b}$ & $0,31 \mathrm{a}$ & 0,35 & 0,33 & 0,32 & 0,30 \\
\hline Aveia + nabo & $0,38 b$ & $0,36 \mathrm{a}$ & $0,35 b$ & 0,33 a & 0,33 & 0,32 & 0,34 & 0,31 \\
\hline Testemunha & $0,33 \mathrm{~b}$ & $0,31 \mathrm{a}$ & $0,35 b$ & $0,32 \mathrm{a}$ & 0,34 & 0,32 & 0,35 & 0,32 \\
\hline SPC & $0,33 \mathrm{~b}$ & $0,26 b$ & $0,35 b$ & $0,28 b$ & 0,36 & 0,31 & 0,36 & 0,31 \\
\hline Mata & $0,49 a$ & $0,39 \mathrm{a}$ & $0,49 \mathrm{a}$ & $0,39 a$ & 0,49 & 0,39 & 0,44 & 0,33 \\
\hline CV\% & 14,02 & 8,18 & 5,78 & 7,38 & 11,06 & 12,78 & 8,37 & 10,85 \\
\hline \multicolumn{9}{|c|}{ Umidade volumétrica $600 \mathrm{KPa}$} \\
\hline Aveia & \multicolumn{2}{|c|}{$0,36 \mathrm{a}$} & \multicolumn{2}{|c|}{$0,30 \mathrm{~ns}$} & \multicolumn{2}{|c|}{$0,29 \mathrm{~ns}$} & \multicolumn{2}{|c|}{$0,30 \mathrm{~ns}$} \\
\hline Centeio & \multicolumn{2}{|c|}{$0,30 \mathrm{~b}$} & \multicolumn{2}{|c|}{0,29} & \multicolumn{2}{|c|}{0,30} & \multicolumn{2}{|c|}{0,30} \\
\hline Nabo & \multicolumn{2}{|c|}{$0,30 \mathrm{~b}$} & \multicolumn{2}{|c|}{0,29} & \multicolumn{2}{|c|}{0,29} & \multicolumn{2}{|c|}{0,30} \\
\hline Centeio + nabo & \multicolumn{2}{|c|}{$0,28 \mathrm{~b}$} & \multicolumn{2}{|c|}{0,29} & \multicolumn{2}{|c|}{0,32} & \multicolumn{2}{|c|}{0,28} \\
\hline Aveia + nabo & \multicolumn{2}{|c|}{$0,32 \mathrm{a}$} & \multicolumn{2}{|c|}{0,29} & \multicolumn{2}{|c|}{0,29} & \multicolumn{2}{|c|}{0,27} \\
\hline Testemunha & \multicolumn{2}{|c|}{$0,28 b$} & & & & & & \\
\hline SPC & & & & & & & & \\
\hline Mata & & & & & & & & \\
\hline CV\% & & & & & & & & \\
\hline
\end{tabular}

Médias seguidas de mesma letra na coluna não diferem pelo teste de Skott-Knott a 5\%; ns: não significativo pelo teste $\mathrm{F}$ a 5\%.

\section{CONCLUSÕES}

O SPDH aumenta os índices de agregação, a porosidade total e umidade volumétrica em comparação ao SPC, e aproxima-se aos índices de agregação em relação à mata.

O nabo solteiro e consorciado com a aveia e o centeio, de maneira geral, melhora os atributos físicos do solo em comparação ao SPC.

\section{AGRADECIMENTOS}

À Chamada MCTI/MAPA/MDA/MEC/MPA/CNPq $\mathrm{N}^{\circ}$ 81/2013 e Edital CHAMADA UNIVERSAL MCTI/CNPq No 14/2014 pelo apoio financeiro e ao $\mathrm{CNPq}$ pelas bolsas de produtividade em pesquisa do primeiro e último autores. 


\section{REFERÊNCIAS BIBLIOGRÁFICAS}

Bronick, C.J. e R. Lal. 2005. Soil structure and management: a review. Geoderma 124, 3-22. Doi: 10.1016/j. geoderma.2004.03.005

Casali, C.A. 2012. Sistemas de culturas sob diferentes manejos em longa duração alteram as formas de fósforo do solo. Tese Doutorado em Ciência do Solo. Universidade Federal de Santa Maria, Santa Maria, Brasil.

COFS-RS/SC. 2004. Manual de adubação e de calagem para os estados para os estados do Rio Grande do Sul e Santa Catarina. 10ª . ed. Comissão de Química e Fertilidade do Solo-RS/SC, Porto Alegre, Brasil.

Epagri. 2013. Sistema de produção para cebola: Santa Catarina. $4^{a}$. revisão. Epagri, Florianópolis, Brasil.

Embrapa. 1997. Manual de métodos de análise de solo. $2^{a}$. ed. Rio de Janeiro, Brasil.

Embrapa. 2013. Sistema brasileiro de classificação de solos. $3^{a}$. ed. Rio de Janeiro, Brasil.

Guedes Filho, O., A.P. Silva, N.F.B. Giarola e C.A.Tormena. 2013. Structural properties of the soil seedbed submitted to mechanical and biological chiseling under no-tillage. Geoderma, 204/205, 94-101. Doi: 10.1016/j. geoderma.2013.04.017

IBGE. 2016. Levantamento sistemático da produção agrícola. IBGE, Rio de Janeiro, Brasil.

Liu, A., B.L. Ma e A.A. Bomke, 2005. Effects of cover crops on soil aggregate stability, total organic carbon, and polysaccharides. Soil Sci. Soc. Am. J. 69, 2041-2048. Doi: 10.2136/sssaj2005.0032

Loss, A., A. Basso, B.S. Oliveira, L.P. Koucher, R.A. Oliveira, C. Kurtz, P.E. Lovato, P. Curmi e G. Brunetto. 2015. Carbono orgânico total e agregação do solo em sistema de plantio direto agroecológico e convencional de cebola. Rev. Bras. Ciênc. Solo, 39, 1212-1224. Doi: 10.1590/01000683rbcs20140718
Luciano, R.V., J.A. Albuquerque, A.L. Mafra, A.Costa e J. Grah. 2014. Water storage variability in a vineyard soil in the southern highlands of Santa Catarina state. Rev. Bras. Ciênc. Solo, 38, 82-93. Doi: 10.1590/ S0100-06832014000100008

Monegat, C. 1991. Manejo de plantas de cobertura do solo em pequenas propriedades. In: Monegat C, editor. Plantas de cobertura do solo: características e manejo em pequenas propriedades. Chapecó: Edição do Autor, Brasil.

Richart, A., J. Tavares Filho, O.R. Brito, R.F. Llanillo e R. Ferreira. 2005. Compactação do solo: causas e efeitos. Semina: Ci. Agrárias, 26, 321-344. Doi: 10.5433/1679-0359.2005v26n3p321

Schoenholtz, S.H.; H. Van Miegroet e J.A. Burger, 2000. A review of chemical and physical properties as indicators of forest soil quality: challenges and opportunities. For. Ecol. Manag. 138, 335-356. Doi: 10.1016/ S0378-1127(00)00423-0

Silva, M.A.S., A.L. Mafra, J.A. Albuquerque, J.D. Rosa, C. Bayer e J. Mielniczuk. 2006. Propriedades físicas e teor de carbono orgânico de um Argissolo Vermelho sob distintos sistemas de uso e manejo. Rev. Bras. Ciênc. Solo, 30, 329-337. Doi: 10.1590/S0100-06832006000200013

Souza, M, J.J. Comin, E.S. Leguizamón, C. Kurtz, G. Brunetto, V. Müller Júnior, B. Ventura, A.P. Camargo. 2013. Matéria seca de plantas de cobertura, produção de cebola e atributos químicos do solo em sistema plantio direto agroecológico. Cienc. Rural, 43, 21-27. Doi: 10.1590/S0103-84782012005000150

Ramos, F.T., D.T. Ramos, M.W. Roque e J.C.S. Maia. 2014. Agregados de um Latossolo Vermelho-Amarelo avaliados por meio de método seco e úmido. Encicl. Biosfera 10, 2935-2950.

Veiga, M. 2011. Metodologia para coleta de amostras e análises físicas do solo. Epagri, Florianópolis, Brasil. 